\title{
Endogenous Retirement and Monetary Cycles ${ }^{1}$
}

\author{
Hippolyte d'ALBIS ${ }^{2}$ \\ Toulouse School of Economics (LERNA) \\ Emmanuelle AUGERAUD-VÉRON ${ }^{3}$ \\ LMA, Université de La Rochelle \\ This version: October 9, 2007 \\ First version: October 8, 2005
}

\footnotetext{
${ }^{1}$ We thank the guest editor Raouf Boucekkine and two anonymous referees for their comments and criticism. We are also grateful to Eric Benoit, Carmen Camacho, Omar Licandro, Pierre Pestieau, Bertrand Wigniolle and the participants of the Florence Vintage Workshop (EUI, 2005), the Toulouse Public Economics Seminar and the OLG Workshop (University of Nantes, 2005) for their comments. The usual disclaimer applies.

${ }^{2}$ Correspondence: LERNA, Université de Toulouse I, 21 allée de Brienne, 31000 Toulouse, France. E-mail: hippolyte.d-albis@univ-tlse1.fr

${ }^{3}$ E-mail: eaugerau@univ-lr.fr
} 


\begin{abstract}
In a model of overlapping generations with a continuum of finitely-lived individuals, the aggregate price dynamics is characterized by a functional differential equation of mixed type. Delays and advances are exogenous when age at retirement is mandatory; they become state-dependent when individuals are allowed to choose their age at retirement. Using the Hopf bifurcation theorem, periodic solutions in the neighborhood of the monetary steady state appearing with a mandatory retirement age vanish with a chosen age.
\end{abstract}

JEL Classification: C62, D91, E3, J26. 


\section{Introduction}

We study the relationship between retirement age and macroeconomic fluctuations and show that allowing individuals to choose their retirement age reduces the volatility of aggregate prices. The proof hinges on the assumption that the economy produces a single non storable good, and on the assumption that the demographic structure is in overlapping generations (OLG) with a continuum of finitely-lived individuals.

The economy has no capital and produces a non storable good using a linear technology with respect to aggregate labor. We also restrict ourselves to monetary equilibria, for which the real value of the aggregate asset holdings remains positive forever. Under mandatory age at retirement, the framework amounts to an exchange economy. Price fluctuations have only nominal and distributional effects. They modify the real consumption at the individual level but not at the aggregate level. When individuals choose their age at retirement, the economy is of the 'yeoman farmer' type. Price fluctuations influence the real aggregate output, and consequently the aggregate consumption.

The second assumption concerns age structure. The OLG model can generate cycles, a feature appealing to economists having business cycles in mind, and linked to sunspot equilibria (Cass and Shell, 1983). However, the first proofs of the existence of such cycles (Gale, 1973; Benhabib and Day, 1982; Grandmont, 1985) relied on an OLG model composed of only two generations. Reichlin (1986), Jullien (1988) and Benhabib and Laroque (1988), who extended the proofs to production economies, made the same assump- 
tion. This is worrying because the cycles these models can generate have periods greater than or equal to the individuals' life-span. Moreover, Sims (1986) conjectured that increasing the frequency of trade among generations would allow individuals to smooth the strong revenue effects necessary for the existence of cycles. The first existence theorem was extended to OLG models with either many generations (Aiyagari, 1989; Reichlin, 1992; Swanson, 1998; Simonovits, 1999; Bhattacharya and Russell, 2003; d'Albis and Augeraud-Véron, 2007) or, using the equivalence argument developed by Balasko et al. (1980), with many commodities (Kehoe and Levine, 1984; Kehoe et al. 1991; Ghiglino and Tvede, 1995). Ghiglino and Tvede (2004) notably prove the existence in a model with many generations and many commodities. However, none of these extensions includes labor supply decisions as in Grandmont (1985). By introducing a choice of age at retirement, we shall test the robustness of these results.

We use a continuous-time OLG model developed by Cass and Yaari (1967), modified to allow for individual retirement decisions as in Boucekkine et al. $(2002,2004)$. The inter-temporal equilibrium is the solution of a nonlinear functional differential equation of mixed type (MFDE). The dynamics is indeed affected by discrete delays and advances. Delays are generated by the vintage structure of the population; advances rely on individuals' expectations. Moreover, when retirement is endogenous, some delays and advances are state-dependent. We characterize the monetary steady state and study the existence of cycles in the neighborhood of the steady state. To prove the existence, we follow Rustichini (1989) by looking for solutions of the linearized MFDE which can have Hopf bifurcation values. We find that for 
certain sets of parameters, a cycle exists when retirement is exogenous; and there is no cycle when retirement is endogenous. This means that the strong revenue effects which still can yield cycles when the frequency of trade is high, are less operative when individuals choose their age at retirement.

In section 2, we present an OLG model with continuous trading. In section 3 we characterize the inter-temporal equilibrium. In section 4 , we study the linearized dynamics in the neighborhood of the monetary steady state.

\section{The Model}

We describe the individual choices and the aggregation procedure. Time $t \geq 0$ is assumed to be continuous and to have a finite starting point.

\subsection{Individual Behavior}

Individuals live $\omega>1$ years. They derive utility from consumption and from the duration of their retirement. Isoelastic preferences and no time discount are assumed. The inter-temporal utility $u(s)$ of an individual born at time $s \geq 0$ is given by:

$$
u(s)=\int_{s}^{s+\omega} \frac{c(s, t)^{1-\frac{1}{\sigma}}}{1-\frac{1}{\sigma}} d t+\frac{\left(\omega-(z(s))^{\alpha}\right)^{1-\frac{1}{\eta}}}{1-\frac{1}{\eta}},
$$

where $c(s, t) \geq 0$ denotes the real consumption of an individual at time $t$ born at time $s$, and $z(s) \in[0, \omega]$ is age of retirement. $\sigma>0$ stands for the elasticity of inter-temporal substitution, $\eta>0$ and $\alpha \in\{0,1\} . \alpha=0$ corresponds to the exogenous retirement case, for which the age at retirement is mandatory and normalized to 1 . When $\alpha=1$, age at retirement is endogenous. 
The real wage $e(s, t)$ is earned only by working individuals:

$$
e(s, t)=\left\{\begin{array}{l}
1 \text { if } t \in\left[s, s+(z(s))^{\alpha}\right] \\
0 \text { otherwise }
\end{array}\right.
$$

Individuals have access to a competitive asset market yielding the interest rate $r(t)$ which is also the deflation rate. $a(s, t)$ is the real wealth of an individual at time $t$ born at time $s$. The instantaneous budget constraint is:

$$
\frac{\partial a(s, t)}{\partial t}=r(t) a(s, t)+e(s, t)-c(s, t) .
$$

Individuals were born with no financial asset and die with no debt. Initial and terminal conditions are $a(s, s)=0$ and $a(s, s+\omega) \geq 0$. We assume that $a(s, t)$ and $c(s, t)$ are piecewise $\mathcal{C}^{1}\left(\mathbb{R}_{+}^{2}\right)$, that $r(t)$ is continuous for all $t \in[s, s+\omega]$ and that $e(s, t)$ is $L^{2}\left(\mathbb{R}_{+}^{2}\right)$. Finally, it will be convenient to define the relative price between time $t$ and time 0 , such that: $R(t)=$ $\exp \left(-\int_{0}^{t} r(u) d u\right)$.

The individual program is to maximize Eq. (1) subject to Eq. (3), under the initial and terminal conditions. The separability of the objective allows us to solve the program in two steps. First, a set of optimal consumption profiles parameterized by $(z(s))^{\alpha}$ is obtained; second, we compute the optimal age at retirement.

Lemma 1 The optimal consumption profile satisfies:

$$
c(s, t)=\frac{\int_{s}^{s+(z(s))^{\alpha}} R(v) d v}{\int_{s}^{s+\omega}(R(v))^{(1-\sigma)} d v}(R(t))^{-\sigma} .
$$

Proof: standard. 
Lemma 2 There exists an optimal age of retirement belonging to $(0, \omega)$ and satisfying:

$$
\alpha R\left(s+(z(s))^{\alpha}\right)\left(\frac{\int_{s}^{s+(z(s))^{\alpha}} R(v) d v}{\int_{s}^{s+\omega}(R(v))^{1-\sigma} d v}\right)^{-\frac{1}{\sigma}}-\alpha\left(\omega-(z(s))^{\alpha}\right)^{-\frac{1}{\eta}}=0 .
$$

The optimal age at retirement is unique if:

$$
-r\left(s+(z(s))^{\alpha}\right)-\frac{1}{\sigma} \frac{R\left(s+(z(s))^{\alpha}\right)}{\int_{s}^{s+(z(s))^{\alpha}} R(v) d v}-\frac{1}{\eta}\left(\omega-(z(s))^{\alpha}\right)^{-1}<0 .
$$

Proof: Replacing Eq. (田) in Eq. (11) yields $\hat{u}(z(s)) . z(s)=0$ is not a solution because $\lim _{z(s) \rightarrow 0} \hat{u}(z(s))=-\infty$ if $\sigma \in(0,1]$ and $\lim _{z(s) \rightarrow 0} \hat{u}^{\prime}(z(s))=$ $+\infty$ if $\sigma>1$. Moreover, $z(s)=\omega$ is not a solution because $\lim _{z(s) \rightarrow \omega} \hat{u}(z(s))=$ $-\infty$ if $\eta \in(0,1]$ and $\lim _{z(s) \rightarrow \omega} \hat{u}^{\prime}(z(s))=-\infty$ if $\eta>1$. Hence, there exists $z(s) \in(0, \omega)$ which is a solution. This optimal solution satisfies $d \hat{u}(z(s)) / d z(s)=0$ or equivalently Eq. (5). Then, a sufficient condition for a global maximum is that $d^{2} \hat{u}(z(s)) / d(z(s))^{2}<0$ at the optimal point. This condition is given by Eq. (6).

The optimal age at retirement is given by a standard consumption-leisure arbitrage. Eq. (4) shows that a longer retirement period implies a lower level of consumption at each age and Eq. (5) shows that the optimum is obtained when the marginal utility yielded by a supplementary unit of leisure equals the marginal dis-utility yielded by the decrease in consumption. Optimal age is necessarily an interior solution of the individual program but one should not exclude multiple local maxima. If the condition given in Eq. (6) is not satisfied, meaning that $r(t)$ is negative, it may indeed exist multiple solutions to Eq. (5). However, in section 4, we shall show that the optimal age of retirement is unique in the neighborhood of the monetary steady state. 


\section{$2.2 \quad$ Aggregation}

The demographic structure is an OLG. Each cohort, whose size is normalized to 1 , is composed of identical individuals. There is no population growth and, at each point of time, a new cohort enters the economy while the oldest one leaves it. The population size is equal to $\omega$.

There exists a single non storable good produced with a linear technology with respect to aggregate labor. The total output $P(t)$ equals the size of the active population, which is solution of:

$$
P(t)=\int_{t-\omega}^{t} e(t-s) d s
$$

where $e(t-s)$ is defined in Eq. (2). The aggregate real consumption $C(t)$ is obtained by integrating over the individual consumptions from their birth dates onward, $c(s, t)$. Replacing Eq. (4) yields:

$$
C(t)=\int_{t-\omega}^{t} \frac{\int_{s}^{s+(z(s))^{\alpha}} R(v) d v}{\int_{s}^{s+\omega}(R(v))^{(1-\sigma)} d v}(R(t))^{-\sigma} d s .
$$

Similarly, the aggregate real wealth is denoted $A(t)$. Money is available; it is a non perishable and non consumable bond which can constitute the counterpart of individual assets. A given quantity of money was distributed at time $t=0$ and there was no other emission since then.

\section{The Monetary Equilibrium}

We prove the existence of the monetary steady state and provide comparative statics. An inter-temporal equilibrium is defined as:

Definition 1 An inter-temporal equilibrium with perfect foresight is a function $F(t)=(C(t), A(t), R(t), P(t), z(t)), F: \mathbb{R}_{+} \rightarrow \mathbb{R}_{+}^{4} \times[0, \omega]$, 
$F(t) \in\left(\mathcal{C}^{1}\left(\mathbb{R}_{+}\right)\right)^{3} \times\left(L^{2}\left(\mathbb{R}_{+}\right)\right)^{2}$ such that (i) individuals maximize their utility subject to budget constraints, (ii) the aggregate consumption equals the output: $C(t)=P(t)$, and (iii) the aggregate wealth is non negative: $A(t) \geq 0$.

The existence of money allows for a positive aggregate wealth at equilibrium. Consider the following particular equilibrium:

Definition 2 A monetary steady state is an inter-temporal equilibrium with perfect foresight such that the aggregate wealth is a positive constant: $A>0$.

Lemma 3 There exists a unique monetary steady state characterized by the quintuple $(C, A, P, R, z)$ which satisfies: $R=1, A=\left(\omega-z^{\alpha}\right) z^{\alpha} / 2, P=C=$ $z^{\alpha}$ where $z^{\alpha}$ is such that:

$$
\alpha\left(\frac{z^{\alpha}}{\omega}\right)^{-\frac{1}{\sigma}}=\alpha\left(\omega-z^{\alpha}\right)^{-\frac{1}{\eta}} .
$$

Proof: At equilibrium, the aggregate consumption equals the output, the aggregate wealth is $d A(t) / d t=r(t) A(t)$. This implies that $r=0$, or equivalently $R=1$, is necessary to obtain a constant and positive aggregate wealth. For $r=0$, the condition in Eq. (6) is satisfied and there exists a unique age of retirement which is constant.

The interest rate equals the demographic growth rate at the monetary steady state (Samuelson, 1958; Gale, 1973). By taking a stationary population, we obtain constant steady state prices and constant individual consumptions over the life cycle. Lemma 3 states that money is always valuated. This is a direct consequence of the introduction of retirement: in OLG economies, money has indeed a value if and only if the average age of the consumer is 
strictly greater than the average age of the worker. Workers finance the pensioners and the aggregate wealth is positive. Given our simple demographic structure, these ages are easy to compute and worth $\omega / 2$ and $z^{\alpha} / 2$ respectively. With the statement of Lemma 2 such that $z^{\alpha} \in(0, \omega)$, we conclude that the monetary steady state exists. In real terms, the value of money is equal to the product of the output and the difference between the average ages of consumption and production. At the limit $z^{\alpha} \rightarrow \omega$, money has no value and the economy is autarkic.

The effect of age at retirement on aggregate wealth is ambiguous. Postponing retirement has a positive effect on wealth if and only if $z^{\alpha}<\omega / 2$. Increasing $z$ increases, on the one hand, the aggregate output and the individual savings while, on the other hand, it increases the average age of the worker which reduces the incentive to save. Another intuition is obtained by computing the age at which each individual begins to dissave. Simple algebra shows that this age is always equal to the age at retirement. Hence, an increase of the age at retirement increases the aggregate wealth if the age at which the dissaving begins belongs to the first half of life.

When age at retirement is exogenous, the monetary steady state is simply characterized by the relative size of the active population. With Lemma 3, aggregate wealth increases with life span $\omega$ : it increases the duration of retirement and consequently creates an additional incentive for individual saving. The elasticity of inter-temporal substitution has no influence on the steady state because the population is stationary; this is no longer true when the age at retirement is endogenous. The following proposition provides some comparative statics for the yeoman farmer economy: 
Proposition 1 When the age at retirement is endogenous,

(i) an increase of longevity increases the age at retirement and aggregate wealth. Moreover, there exists $\bar{\omega}>1$ such that $z \geq 1 \Leftrightarrow \omega \geq \bar{\omega}$.

(ii) an increase of the elasticity of inter-temporal substitution reduces the age at retirement and has an ambiguous effect on the aggregate wealth. Moreover, if $\omega<2$, there exists $\bar{\sigma}>0$ such that $z \geq 1 \Leftrightarrow \sigma \leq \bar{\sigma}$; if $\omega \geq 2, z>1$.

Proof: For $\alpha=1$, use Eq. (9) as an implicit equation. For part (i), $d z / d \omega \in(0,1)$ and $d A / d \omega>0$. Finally, $\bar{\omega}$ is computed explicitly by replacing $z=1$ in the implicit equation. For part (ii), because $\omega-z<1$ is always true, one has $d z / d \sigma<0$. Hence, the sign of $d A / d \sigma$ is the opposite of the one of $d A / d z$ if and only if $z>\omega / 2$. Finally, with the implicit equation, $z \rightarrow \omega$ when $\sigma \rightarrow 0$ and, replacing $z=1$ compute $\bar{\sigma}$ to conclude that $\bar{\sigma}$ is positive only if $\omega<2$.

The intuition for Proposition 1 is that because of the consumption/leisure arbitrage, an increase in longevity increases both the age and the duration of retirement; hence, $d z / d \omega \in(0,1)$. The magnitude of the latter derivative depends on the parameter $\eta$ characterizing the curvature of the utility function with respect to leisure. A lower $\eta$ means a utility more concave and a higher $d z / d \omega$. At the limit $\eta \rightarrow 0$, one obtains an age at retirement that goes to its lower bound: $\omega-1$, and $d z / d \omega \rightarrow 1$. The effect of longevity on aggregate wealth is ambiguous because it increases both the average age of the consumer and the average age of the worker. However, as $d z / d \omega \in(0,1)$, the final effect is always positive. Chang (1991) and Kalemli-Ozcan and Weil (2004) pointed out the importance of the assumption of certainty on indi- 
vidual life span. In case of uncertain life span, an increase in longevity can reduce the age at retirement.

The effect of an increase of the elasticity of inter-temporal substitution on retirement is negative. Indeed, at the monetary steady state where the interest rate is zero, the elasticity does not influence the individual consumption growth rate. It only modifies the arbitrage between consumption and leisure in favor of the second one, which ultimately means less human wealth and less consumption. The effect of the elasticity on aggregate wealth is the opposite of the effect of the age at retirement on wealth.

\section{Monetary Cycles}

In the linearized dynamics in the neighborhood of the monetary steady state given by Lemma 3, we analyze exogenous and endogenous retirement and look for particular long-run fluctuations.

Definition 3 A monetary cycle is a periodic solution of the intertemporal equilibrium in the vicinity of the monetary steady state.

\subsection{Exogenous Retirement}

When the age at retirement is mandatory, replacing (27) in (7) yields the size of the active population: $P(t)=1$ for all $t$. The inter-temporal equilibrium is characterized by the functional differential equation:

$$
(R(t))^{\sigma}=\int_{t-\omega}^{t} \frac{\int_{s}^{s+1} R(v) d v}{\int_{s}^{s+\omega}(R(v))^{(1-\sigma)} d v} d s .
$$

The dynamics of $R(t)$ depends on the entire set of observations of $R$ in the interval $[t-\omega, t+\omega]$. Past observations, bringing delays in the dynamics, 
are generated by the vintage structure of human capital as in Boucekkine, de la Croix and Licandro (2002), while future observations, yielding advances in the dynamics, come from perfect foresight. Eq. (10) is non linear for any $\sigma \neq 1$, meaning that revenue and substitution effects do not counterbalance each other.

Following Rustichini (1989), the proof of the existence of periodic solutions uses the Hopf bifurcation theorem. We consider the local dynamics around $R=1$ : it is the one of $x(t)$ defined such that $R(t)=1+\varepsilon x(t)$.

Property 1 The characteristic function $H(\lambda)$ of $x(t)$ satisfies:

$$
H(\lambda)=\int_{-\omega}^{0}\left(\int_{s}^{s+1} e^{\lambda v} d v\right) d s-\omega \sigma-\frac{(1-\sigma)}{\omega} \int_{-\omega}^{0}\left(\int_{s}^{s+\omega} e^{\lambda v} d v\right) d s .
$$

Proof: Replace $R(t)=1+\varepsilon x(t)$ in Eq. (10) and Taylor expand in the neighborhood of $\varepsilon=0 . H(\lambda)$ is obtained by the change of variable $x(t)=e^{\lambda t}$ and some algebra.

The characteristic function $H(\lambda)$ has an infinity of complex roots with negative real parts and an infinity of complex roots with positive real parts. This implies that the linearized dynamics is initially characterized by oscillations that eventually disappear. These fluctuations are of few interest because we study the dynamics in the neighborhood of the steady state. The following lemma focuses on permanent fluctuations yielded by the pure imaginary roots of $H(\lambda)$.

Lemma 4 There exist $(\omega, \sigma)$ such that $H(\lambda)$ has pure imaginary roots which are Hopf bifurcation values.

Proof: 1) we assume $\lambda=i q$ and prove that there exists a least one $q>0$ such that $H(i q)=0$. We define $\left(\omega_{0}, \sigma_{0}\left(\omega_{0}\right)\right)$ the pair of parameters for which 
such a root exists. 2) We use $\sigma$ as a bifurcation parameter and show that there exists a neighborhood of $\sigma_{0}$ such that $d \operatorname{Re}(H(\lambda)) / d \sigma$ is not equal to zero while $d \operatorname{Im}(H(\lambda)) / d \sigma=0$.

1) Replace $\lambda=i q$ in Eq. (11) to obtain $H(i q)=\operatorname{Re}(H(i q))+i \operatorname{Im}(H(i q))$ with:

$$
\begin{aligned}
\operatorname{Re}(H(i q))= & -\left(\frac{\cos (q)-1-\cos (q(\omega-1))+\cos (q \omega)}{q^{2}}\right) \\
& -\omega \sigma+\frac{2(1-\sigma)}{\omega}\left(\frac{\cos (q \omega)-1}{q^{2}}\right), \\
\operatorname{Im}(H(i q))= & -\left(\frac{\sin (q)+\sin (q(\omega-1))-\sin (q \omega)}{q^{2}}\right) .
\end{aligned}
$$

(i) We show that there exist, for any $\sigma$, some $\left(\omega_{0}, q\left(\omega_{0}\right)\right)$ such that $\operatorname{Im}\left(H\left(i q\left(\omega_{0}\right)\right)\right)=$

0 . (ii) it shows that $\operatorname{Re}\left(H\left(i q\left(\omega_{0}\right)\right)\right)=0$ is compatible with some $\sigma>0$.

(i) Eq. (13) is also:

$$
\operatorname{Im}(H(i q))=-\frac{4 \sin \left(\frac{q(\omega-1)}{2}\right) \sin \left(\frac{q}{2}\right) \sin \left(\frac{q \omega}{2}\right)}{q^{2} \omega} .
$$

Roots of $\operatorname{Im}(H(i q))$ are then $q=2 k \pi, q=2 k \pi / \omega$ and $q=2 k \pi /(\omega-1)$ for $k \in \mathbb{Z}$. Eq. $\operatorname{Re}(H(i q))=0$ is rewritten as:

$$
\sigma=\frac{-4 \sin ^{2}\left(\frac{q \omega}{2}\right)+2 \omega\left[\sin ^{2}\left(\frac{q \omega}{2}\right)+\sin ^{2}\left(\frac{q}{2}\right)\right]-\omega+\omega \cos (q(\omega-1))}{-4 \sin ^{2}\left(\frac{q \omega}{2}\right)+(\omega q)^{2}} .
$$

Consider the roots $q=2 k \pi /(\omega-1)$ for $k \in \mathbb{Z}$, replace them in Eq. (15) and rearrange to obtain:

$$
\sigma=\frac{(\omega-1)}{\frac{(q \omega)^{2}}{4 \sin ^{2}\left(\frac{q}{2}\right)}-1} .
$$

With $\omega>1$, conclude that the RHS of Eq. (16) is positive. For any $(\omega, 2 k \pi /(\omega-1)), k \in \mathbb{Z}, \operatorname{Im}(H(i q))=0$ is satisfied and there exists a $\sigma>0$ such that $\operatorname{Re}(H(i q))=0$. Notice that $\operatorname{Re}(H(i q)) \neq 0$ for $q=2 k \pi$. 
2) Let $\lambda=p+i q$. Using Eq. (11), one has:

$$
\frac{d \operatorname{Re}(H(\lambda))}{d \sigma}=-\omega+\frac{\cos (q \omega)}{\omega} \int_{-\omega}^{0}\left(\int_{s}^{s+\omega} e^{p v} d v\right) d s .
$$

Compute Eq. (匹7) for $p=0$, it is not zero because $q \neq 2 k \pi / \omega$.

The following lemma indicates the space of parameters giving rise to a cycle.

Lemma 5 There exists a monetary cycle of period $q$ only if $\sigma<q / \omega$.

Proof: We prove the lemma for $\omega<2$, which is the realistic case for a age at retirement equal to 1 . The proof for $\omega \geq 2$ is similar and available upon request. We shall show that $\sigma-q / \omega<0$ when $\sigma$ is defined by Eq. (16) and $q=2 k \pi /(\omega-1)$. First, $\sigma-q / \omega<\phi(k, \omega)$ with

$$
\phi(k, \omega)=\frac{(\omega-1)}{\left(\frac{\omega k \pi}{(\omega-1)}\right)^{2}-1}-\frac{2 k \pi}{\omega(\omega-1)} .
$$

It is sufficient to show that $\phi(k, \omega)<0$. Because $\partial \phi(k, \omega) / \partial k<0$, we have to prove that $\phi(1, \omega)<0$. Then, $\partial \phi(1, \omega) / \partial \omega>0$ for $\omega>1$ and that $\phi(1,2) \simeq-3$.

Sufficiently strong revenue effects are necessary to obtain monetary cycles. The magnitude of these effects depends on the periodicity of the price cycle or, equivalently, of the inflation rate cycle, with respect to the individual lifespan: to obtain a cycle with a lower periodicity, a stronger revenue effect is necessary. We obtain this result with a discount rate equal to zero although cycles are more likely to occur when individuals discount the future. 


\subsection{Endogenous Retirement}

To identify the inter-temporal equilibrium, we characterize the active population size and how cohorts leave the labor market, and we define the "last in, last out" property.

Definition 4 For all $t \geq 0$, let $s_{0}$ be the greatest $s \in[t-\omega, t]$ such that $s_{0}+z\left(s_{0}\right)=t$. Cohorts satisfy the "last in, last out" property if and only if for all $s<s_{0}, s+z(s) \geq t$.

According to definition 4, when there is "last in, last out", cohorts leave the labor market in the same order they have entered it; hence $P(t)=$ $z(t-P(t))$. Otherwise, it can exist a date $t_{0}$ such that there is no cohort leaving the labor market and a date $t_{1}$ such that different cohorts leave it simultaneously. In such situations, the analytical characterization of the inter-temporal equilibrium would be complicate. However, the next lemma excludes such situations in the neighborhood of the steady state.

Lemma 6 In the neighborhood of the monetary steady state, the "last in, last out" property holds.

Proof: The optimal age at retirement, defined as the $z($.$) solution of$ Eq. (5), is continuously differentiable with respect to $s$. Indeed, as $R(.) \in$ $C^{1}\left(\mathbb{R}_{+}\right)$, then $z(s)$ is $C^{1}\left(\mathbb{R}_{+}\right)$. Consequently, there is "last in, last out" if and only if $1+d z(s) / d s>0$. In the neighborhood of the monetary steady state this condition is satisfied because $d z(s) / d s=0$.

It is now possible to characterize the inter-temporal equilibrium in the neighborhood of the monetary steady state. It is the solution of the following system of non linear functional differential equations with state-dependent 
delays and advances:

$$
\left\{\begin{array}{l}
P(t)(R(t))^{\sigma}=\int_{t-\omega}^{t}(R(s+z(s)))^{\sigma}(\omega-z(s))^{\frac{\sigma}{\eta}} d s \\
\int_{t}^{t+z(t)} R(v) d v=(R(t+z(t)))^{\sigma}(\omega-z(t))^{\frac{\sigma}{\eta}} \int_{t}^{t+\omega}(R(v))^{1-\sigma} d v \\
P(t)=z(t-P(t)) .
\end{array}\right.
$$

The first equation of System (19) is the equilibrium condition in the good market which was modified by replacing the optimal condition on individual retirement (the second equation). The dynamics of $R$ is governed by distributed delays and advances while the dynamics of $z$ depends on the future observations of $R$ only. The third equation characterizes the population size when there is "last in, last out". The main difference between system (19) and Eq. (10) consists of the presence of state-dependent delays and advances.

Consider the local dynamics around the steady state defined by Eq. (9). After Cooke and Huang (1996), it is the one of $(x(t), y(t), h(t))$ defined such that:

$$
\left\{\begin{array}{l}
R(t)=1+\varepsilon x(t) \\
z(t)=z+\varepsilon y(t) \\
P(t)=z+\varepsilon h(t)
\end{array}\right.
$$

where $z$ satisfies $\left(\frac{z}{\omega}\right)^{-\frac{1}{\sigma}}-(\omega-z)^{-\frac{1}{\eta}}=0$. State-dependent delays and advances vanish in the linearized system and it is possible to apply Rustichini (1989).

Property 2 The characteristic function of the linearized system is denoted $Q(\lambda)$ and satisfies:

$$
\begin{aligned}
Q(\lambda)= & 2 \omega+\frac{\sigma z \omega}{\eta(\omega-z)}+\frac{(\omega-z) \eta \omega}{\sigma z}-\left(1+\frac{\sigma z}{(\omega-z) \eta \omega} \int_{-\omega}^{0} e^{\lambda(s+z)} d s\right) \\
& *\left(\frac{(\omega-z) \eta \omega}{\sigma z}+\frac{\omega}{z \sigma} \int_{0}^{z} e^{\lambda(v-z)} d v-\frac{(1-\sigma)}{\sigma} \int_{0}^{\omega} e^{\lambda(v-z)} d v\right) \cdot(21)
\end{aligned}
$$

Proof: Replace system (20) in system (19) and Taylor expand in the neighborhood of $\varepsilon=0$. This yields $h(t)=y(t-z)$ and a system of two 
equations. Write the Jacobian matrix $\mathbf{J}$ and then $Q(\lambda)=\operatorname{det} \mathbf{J}$. Some algebra yields Eq. (21).

As in the previous case, only pure imaginary roots are considered.

Lemma $7 Q(\lambda)$ has no pure imaginary roots.

Proof: The proof shows that $|Q(i q)|>0$. Using Eq. (21), one has:

$$
\begin{aligned}
|Q(i q)|= & \left|2 \omega+\frac{\sigma z \omega}{\eta(\omega-z)}+\frac{(\omega-z) \eta \omega}{\sigma z}-\right|\left(1+\frac{\sigma z}{(\omega-z) \eta \omega} \int_{-\omega}^{0} e^{i q(s+z)} d s\right) \\
& *\left(\frac{(\omega-z) \eta \omega}{\sigma z}+\frac{\omega}{z \sigma} \int_{0}^{z} e^{i q(v-z)} d v-\frac{(1-\sigma)}{\sigma} \int_{0}^{\omega} e^{i q(v-z)} d v\right) \| .(22)
\end{aligned}
$$

Consequently, $|Q(i q)| \geq|\phi(\sigma)|$ where:

$$
\begin{aligned}
\phi(\sigma)= & 2 \omega+\frac{\sigma z \omega}{\eta(\omega-z)}+\frac{(\omega-z) \eta \omega}{\sigma z} \\
& -\frac{\omega}{\sigma}\left(1+\frac{\sigma z}{(\omega-z) \eta}\right)\left(\frac{(\omega-z) \eta}{z}+(1+\varepsilon)+|1-\sigma|\right) .
\end{aligned}
$$

with $\varepsilon>0$. Showing that $\phi(\sigma)<0$ for all $\sigma>0$ is then sufficient to conclude. One indeed has: $\phi^{\prime}(\sigma)>0$ while $\phi(1)<0$ and $\lim _{\sigma \rightarrow+\infty} \phi(\sigma)<0$.

The main result of this section is presented in:

Proposition 2 When the age at retirement is optimally chosen by individuals, the occurrence of monetary cycles is ruled out.

Proof: Given lemmas 1 and 7, the proof is immediate.

We now turn to the general comment of this section. In the exogenous retirement case, we have shown that increasing the frequency of trade in generations is not sufficient to smooth the strong revenue effects which can yield cycles in OLG models. Conversely, introducing a simple leisure choice modelled as a retirement decision was proved to be necessary for the occurrence 
of monetary cycles. The intuition is that for mandatory age at retirement, an anticipation of high prices, corresponding to a low interest rate, increases the individual savings when the elasticity of inter-temporal substitution is small. This increases the aggregate wealth and increases the prices. But if individuals are allowed to choose their age at retirement, an anticipation of high prices is an incitation to retire later because high prices imply high nominal wages. This lowers the incitation to save and the final effect on aggregate wealth is lower than in the mandatory retirement case.

Proposition 2 should extend to more general economies. First, allowing for endogenous entrance into the labor market, with a schooling decision as in de la Croix and Licandro (1999), would produce the same result: cycles would be more likely with a mandatory age for the end of education than with an optimally chosen one. In production economies, some non linearities are added, and then endogenous cycles are possible even with endogenous labor supply. This was notably shown by Whitesell (1986) and Matsuyama (2005). However, in a two-period framework, Cazzavillan and Pintus (2004) and Nourry and Venditti (2006) proved that cycles occur less frequently when individuals consume during their youth.

\section{Conclusion}

We analyzed the existence of long-run fluctuations in OLG economies. We showed that when individuals choose their age at retirement, certain periodic solutions of the inter-temporal equilibrium dynamics vanish. The study of the existence and uniqueness of the inter-temporal equilibrium remains to be done. For linear MFDE, the existence problem was studied by d'Albis 
and Augeraud-Véron (2004) while the indeterminacy issue was analyzed by Demichelis and Polemarchakis (2007). However, the problem is heightened with state-dependent delays because of the characterization of the initial conditions.

\section{References}

[1] Aiyagari, S. R. (1989). Can there be short-period deterministic cycles when people are long lived? Quarterly Journal of Economics, 104: 163185.

[2] Albis (d'), H., and Augeraud-Véron, E. (2004). Competitive growth in a life-cycle model: existence and dynamics. International Economic Review, forthcoming.

[3] Albis (d'), H., and Augeraud-Véron, E. (2007). Balanced cycles in an OLG model with a continuum of finitely-lived individuals. Economic Theory, 30: 181-186.

[4] Balasko, Y., Cass, D., and Shell, K. (1980). Existence of competitive equilibrium in a general overlapping generations model. Journal of Economic Theory, 23: 307-322.

[5] Bhattacharya, J., and Russell, S. (2003). Two-period cycles in a threeperiod overlapping generations model. Journal of Economic Theory, 109: $378-401$.

[6] Benhabib, J., and Day, R. H. (1982). A characterization of erratic dynamics in the overlapping generations model. Journal of Economic Dynamics and Control, 4: 37-55.

[7] Benhabib, J., and Laroque, G. (1988). On competitive cycles in productive economies. Journal of Economic Theory, 45: 145-170.

[8] Boucekkine, R., de la Croix, D., and Licandro, O. (2002). Vintage human capital, demographic trends and endogenous growth. Journal of Economic Theory, 104: 340-375.

[9] Boucekkine, R., de la Croix, D., and Licandro, O. (2004). Modelling vintage structures with DDEs: Principles and applications. Mathematical Population Studies, 11: 151-179.

[10] Cass, D., and Shell, K. (1983). Do sunspots matter? Journal of Political Economy, 91: 193-227. 
[11] Cass, D., and Yaari, M. E. (1967). Individual saving, aggregate capital accumulation, and efficient growth, in Shell, K.: Essays on the Theory of Optimal Economic Growth, pp. 233-268, Cambridge, MA: MIT Press.

[12] Cazzavillan G., and Pintus, P. A. (2004). Robustness of multiple equilibria in OLG economies. Review of Economic Dynamics, 7: 456-475.

[13] Chang, F-R. (1991). Uncertain lifetimes, retirement and economic welfare. Economica, 58: 215-232.

[14] Cooke, K. L., and Huang, W. (1996). On the problem of linearization for state-dependent delay differential equations. Proceedings of the American Mathematical Society, 124: 1417-1426.

[15] de la Croix, D., and Licandro, O. (1999). Life expectancy and endogenous growth. Economics Letters, 65: 255-263.

[16] Demichelis, S., and Polemarchakis, H. (2007). The determinacy of equilibrium in economies of overlapping generations. Economic Theory, 32: 461-475.

[17] Gale, D. (1973). Pure exchange equilibrium of dynamic economic models. Journal of Economic Theory, 6: 12-36.

[18] Ghiglino, C., and Tvede, M. (1995). Endowments, stability, and fluctuations in OG models, Journal of Economic Dynamics and Control, 19: 621-653.

[19] Ghiglino, C., and Tvede, M. (2004). Dynamics in OG economies, Journal of Difference Equations and Applications, 10: 463-472.

[20] Grandmont, J-M. (1985). On endogenous competitive business cycles. Econometrica, 53: 995-1045.

[21] Jullien, B. (1988). Competitive business cycles in an overlapping generations economy with productive investment. Journal of Economic Theory, 46: $45-65$.

[22] Kalemli-Ozcan, S., and Weil, D. (2004). Mortality change, the uncertainty effect and retirement. Working paper, Brown University.

[23] Kehoe, T. J., and Levine, D. K. (1984). Regularity in overlappinggenerations exchange economies. Journal of Mathematical Economics, 13: $69-93$

[24] Kehoe, T. J., Levine, D. K., Mas-Colell, A., and Woodford, M. (1991). Gross substitutability in large-square economies. Journal of Economic Theory, 54: 1-25.

[25] Matsuyama, K. (2005). A one-sector neoclassical growth model with endogenous retirement. Japanese Economic Review, forthcoming. 
[26] Nourry, C., and Venditti, A. (2006). Overlapping generations model with endogenous labor supply: general formulation. Journal of Optimization Theory and Applications, 128: 355-377.

[27] Reichlin, P. (1986). Equilibrium cycles in an overlapping generations economy with production. Journal of Economic Theory, 40: 89-102.

[28] Reichlin, P. (1992). Endogenous cycles with long-lived agents. Journal of Economic Dynamics and Control, 16: 243-266.

[29] Rustichini, A. (1989). Hopf bifurcation for functional differential equations of the mixed type. Journal of Dynamics and Differential Equations, 1: $145-177$.

[30] Samuelson, P. A. (1958). An exact consumption-loan model of interest with or without the social contrivance of money. Journal of Political Economy, 66: 467-482.

[31] Simonovits, A. (1999). Are there cycles in realistic overlapping cohorts models? Structural Change and Economic Dynamics, 10: 261-275.

[32] Sims, C. A. (1986). Comments, in Sonnenschein, H. F.: Models of Economic Dynamics, pp. 37-39, New York: Springer.

[33] Swanson, C. E. (1998). A volatility bound for endogenous business cycles: the case of even period lives with additive utility in a monetary economy. Economics Letters, 61: 339-344.

[34] Whitesell, W. (1986) Endogenous cycles with uncertain life-spans in continuous time. Economic Letters, 22: 153-158. 